\title{
ESSENTIAL DIMENSION OF ALGEBRAIC TORI
}

\author{
ROLAND LÖTSCHER ${ }^{(1)}$, MARK MACDONALD, AUREL MEYER ${ }^{(2)}$, \\ AND ZINOVY REICHSTEIN ${ }^{(3)}$
}

\begin{abstract}
The essential dimension is a numerical invariant of an algebraic group $G$ which may be thought of as a measure of complexity of $G$-torsors over fields. A recent theorem of N. Karpenko and A. Merkurjev gives a simple formula for the essential dimension of a finite $p$-group. We obtain similar formulas for the essential $p$-dimension of a broad class of groups, which includes all algebraic tori.
\end{abstract}

\section{INTRODUCTION}

Throughout this paper $p$ will denote a prime integer, $k$ an arbitrary base field and $G$ a (not necessarily smooth) algebraic group defined over $k$. Unless otherwise specified, all fields are assumed to contain $k$ and all morphisms between them are assumed to be $k$-homomorphisms. Morphisms of algebraic groups over $k$ are assumed to be defined over $k$.

Let $K$ be a field and $H^{1}(K, G)$ be the nonabelian cohomology set with respect to the finitely presented faithfully flat (fppf) topology. Equivalently $H^{1}(K, G)$ is the set of isomorphism classes of $G$-torsors over $\operatorname{Spec}(K)$. If $G$ is smooth then one may identify $H^{1}(*, G)$ with the first Galois cohomology functor. We say that $\alpha \in H^{1}(K, G)$ descends to an intermediate field $k \subset$ $K_{0} \subset K$ if it lies in the image of the natural map $H^{1}\left(K_{0}, G\right) \rightarrow H^{1}(K, G)$. The minimal transcendence degree $\operatorname{trdeg}_{k}\left(K_{0}\right)$, where $\alpha$ descends to $K_{0}$, is called the essential dimension of $\alpha$ and is denoted by the symbol ed $(\alpha)$. The essential dimension of the group $G$ is the supremum of ed( $\alpha)$, as $K$ ranges over all field extensions of $k$ and $\alpha$ ranges over $H^{1}(K, G)$. This numerical invariant of $G$ has been extensively studied in recent years; see $\mathrm{BF}, \mathrm{BR}, \mathrm{Re}$, $\mathrm{RY}, \mathrm{Me}_{1}$.

For many groups $G$ the essential dimension $\operatorname{ed}(G)$ is hard to compute, even over the field $k=\mathbb{C}$ of complex numbers. Given a prime $p$, it is often

2000 Mathematics Subject Classification. 20G15, 11E72, 20C10.

Key words and phrases. Essential dimension, group of multiplicative type, algebraic torus, twisted finite group, lattice, Galois module.

(1) Roland Lötscher was partially supported by the Swiss National Science Foundation (Schweizerischer Nationalfonds).

(2) Aurel Meyer was partially supported by a University Graduate Fellowship at the University of British Columbia.

(3) Zinovy Reichstein was partially supported by NSERC Discovery and Accelerator Supplement grants. 
easier to compute the essential $p$-dimension, $\operatorname{ed}(G ; p)$, which is defined as follows. The essential $p$-dimension ed $(\alpha ; p)$ of $\alpha \in H^{1}(K, G)$ is the minimal value of ed $\left(\alpha_{L}\right)$, as $L$ ranges over all finite field extensions of $K$ of degree prime to $p$. The essential $p$-dimension $\operatorname{ed}(G ; p)$ of $G$ is then the supremum of ed $(\alpha ; p)$ taken over all fields $K$ containing $k$ and all $\alpha \in H^{1}(K, G)$. For details on this notion, see $[\mathrm{RY}]$ or $\left[\mathrm{Me}_{1}\right.$. Clearly $0 \leq \operatorname{ed}(G ; p) \leq \operatorname{ed}(G)$. It is also easy to check that if $L / K$ is a finite extension of degree prime to $p$ then

$$
\operatorname{ed}(G ; p)=\operatorname{ed}\left(G_{L} ; p\right)
$$

see $\mathrm{Me}_{1}$, Proposition 1.5].

A representation $\psi: G \rightarrow \mathrm{GL}(V)$ is called generically free if there exists a non-empty $G$-invariant open subset $U \subset V$ such that the scheme-theoretic stabilizer of every point of $U\left(k_{\text {alg }}\right)$ is trivial. Such a representation gives rise to an upper bound on the essential dimension,

$$
\operatorname{ed}(G ; p) \leq \operatorname{ed}(G) \leq \operatorname{dim}(V)-\operatorname{dim}(G) ;
$$

see [Me1, Theorem 4.1], [Re, Theorem 3.4], [BF, Lemma 4.11].

N. Karpenko and A. Merkurjev [KM] recently showed that the inequalities (21) are in fact sharp for finite constant $p$-groups, assuming that the base field $k$ contains a primitive $p$ th root of unity (note that this implies char $k \neq p)$. The purpose of this paper is to establish a similar result for a large class of groups which includes all algebraic tori.

For a field extension $l / k$, set $G_{l}:=G \times_{\operatorname{Spec} k} \operatorname{Spec}(l)$. Let $k_{\text {sep }}$ be a fixed separable closure of $k$. Recall that an algebraic group $G$ over a field $k$ is called diagonalizable if it isomorphic to a closed subgroup of $\mathbb{G}_{m}^{n}$ for some $n \geq 0 ; G$ is said to be of multiplicative type if $G_{k_{\text {sep }}}$ is diagonalizable, see, e.g., $\mathrm{Vos}_{2}$, Section 3.4]. Smooth connected groups of multiplicative type are precisely the algebraic tori.

Recall that the order of an algebraic group $F$ is defined as $|F|=\operatorname{dim}_{k} k[F]$; algebraic groups of finite order are called finite. We will say that a representation $\psi: G \rightarrow \mathrm{GL}(V)$ of an algebraic group $G$ is $p$-faithful if its kernel is finite and of order prime to $p$.

Theorem 1.1. Let $G$ be a group of multiplicative type over an arbitrary field $k$. Assume that $G$ has a Galois splitting field of p-power degree. Then

$$
\operatorname{ed}(G ; p)=\min \operatorname{dim}(\psi)-\operatorname{dim} G,
$$

where the minimum is taken over all p-faithful representations $\psi$ of $G$. Moreover, if $G$ is an extension of a p-group by a torus then

$$
\operatorname{ed}(G)=\operatorname{ed}(G ; p) .
$$

The quantity $\min \operatorname{dim}(\psi)$ which appears in the statement of the Theorem 1.1 can be conveniently described in terms of character modules; see Corollary [5.1. We give several applications of these results in Sections 5 and 6. Further applications of the Theorem 1.1, to the classical problem 
of computing essential dimensions of central simple algebras, can be found in $\mathrm{Me}_{2}$ and $[\mathrm{BM}$.

Note that Theorem 1.1 allows us to compute $\operatorname{ed}(G ; p)$ for any group $G$ of multiplicative type over $k$. Indeed, we can always choose a finite field extension $k^{\prime} / k$ of degree prime to $p$ such that $G_{k^{\prime}}$ has a Galois splitting field of $p$-power degree. In view of (11), $\operatorname{ed}(G ; p)=\operatorname{ed}\left(G_{k^{\prime}} ; p\right)$, and the latter number is given by Theorem 1.1 .

In the last section we will prove analogous results for a finite (not necessarily abelian) algebraic group over $k$, assuming char $k \neq p$; see Theorem 7.1 and Remark 7.2

\section{Preliminaries on Groups of multiplicative type}

Throughout this section, $A$ will denote an algebraic group of multiplicative type over a field $k, X(A)$ the character group of $A$, and $\Gamma:=\operatorname{Gal}\left(k_{\mathrm{sep}} / k\right)$ the absolute Galois group of $k$. Then $X(A)$ is a continuous $\mathbb{Z} \Gamma$-module. Moreover, $X(*)$ defines an anti-equivalence between algebraic $k$-groups of multiplicative type and continuous $\mathbb{Z} \Gamma$-modules; see, e.g., [Wa, 7.3]. Let Diag denote the inverse of $X$, so that $\operatorname{Diag}(X(A)) \simeq A$.

Given a field extension $l / k$, recall that $A$ is called split over $l$ if and only if the absolute Galois group $\operatorname{Gal}\left(l_{\mathrm{sep}} / l\right)$ acts trivially on $X(A)$. If a torsionfree $\mathbb{Z} \Gamma$-module $P$ has a basis which is permuted by $\Gamma$, then it is called a permutation module, and $\operatorname{Diag}(P)$ is a quasi-split torus.

We will write $A[p]$ for the $p$-torsion subgroup $\left\{a \in A \mid a^{p}=1\right\}$ of $A$. Clearly $A[p]$ is defined over $k$. If $A$ is a finite algebraic group of multiplicative type, then $|A|=|X(A)|$ (by Cartier duality).

It is well known how to construct a maximal split subtorus of an algebraic torus, see for example [Wa, 7.4]. The following is a variant of this construction for algebraic groups of multiplicative type. Set

$$
\operatorname{Split}_{k}(A):=\operatorname{Diag}\left(X(A)_{\Gamma}\right)
$$

where $X(A)_{\Gamma}$ is the module of co-invariants, defined as the largest quotient of $X(A)$ with trivial $\Gamma$-action. Clearly $\operatorname{Split}_{k}(A)$ is split over $k$.

Lemma 2.1. If $A[p] \neq\{1\}$ and $A$ is split over a Galois extension $l / k$ of $p$-power degree, then $\operatorname{Split}_{k}(A) \neq\{1\}$.

Proof. If $B$ is a $k$-subgroup of $A$ then $\operatorname{Split}_{k}(B) \subset \operatorname{Split}_{k}(A)$, so it suffices to show that $\operatorname{Split}_{k}(A[p]) \neq\{1\}$. Hence, we may assume that $A=A[p]$ or equivalently, that $X(A)$ is a finite-dimensional $\mathbb{F}_{p}$-vector space on which the $p$-group $\operatorname{Gal}(l / k)$ acts. Any such action is upper-triangular, relative to some $\mathbb{F}_{p}$-basis $e_{1}, \ldots, e_{n}$ of $X(A)$; see, e.g., $\mathrm{Se}_{1}$, Proposition 26, p. 64]. That is,

$$
\gamma\left(e_{i}\right)=e_{i}+\left(\mathbb{F}_{p^{-}} \text {linear combination of } e_{i+1}, \ldots, e_{n}\right)
$$


for every $i=1, \ldots, n$ and every $\gamma \in \operatorname{Gal}(l / k)$. The quotient of $X(A)$ by the linear span of $e_{2}, \ldots, e_{n}$ has trivial $\Gamma$-action. Hence the module of coinvariants $X(A)_{\Gamma}$ is non-trivial. Then $\operatorname{Split}_{k}(A)=\operatorname{Diag}\left(X(A)_{\Gamma}\right)$ is nontrivial as well.

Let $G$ be an algebraic group whose centre $Z(G)$ is of multiplicative type. Then we define $C(G):=\operatorname{Split}_{k}(Z(G)[p])$. Note that this definition depends on the prime $p$, which we assume to be fixed throughout.

Lemma 2.2. Let $N$ be a subgroup of $A$ defined over $k$. Assume that $A$ has a Galois splitting field $l / k$ of $p$-power degree. Then $N \cap C(A)=\{1\}$ if and only if $N$ is finite and its order is prime to $p$.

Proof. If the order of $N \subseteq A$ is finite and prime to $p$ then clearly $N \cap C(A)=$ $\{1\}$, because $C(A)$ is a $p$-group. Conversely, suppose the order of $N$ is either infinite or is finite but divisible by $p$. Then $N[p] \neq\{1\}$, and $N[p]$ is split by $l$. By Lemma 2.1, $\{1\} \neq \operatorname{Split}_{k}(N[p]) \subseteq \operatorname{Split}_{k}(A[p])=C(A)$, as desired.

Now suppose $l / k$ be a Galois splitting field of $A$ and $\psi: A \rightarrow \mathrm{GL}(V)$ is a $k$ representation. Then we can decompose $V_{l}=\bigoplus_{\chi \in \Lambda} V(\chi)$, where $\Lambda \subseteq X(A)$ is the set of weights and $V(\chi) \subset V$ is the weight space associated to $\chi \in \Lambda$, i.e., the subspace of $V$, where $A$ acts via $\chi$. The Galois group $\Gamma=\operatorname{Gal}(l / k)$ permutes $\Lambda$ and weight spaces $V(\chi)$.

Lemma 2.3. Let $d_{\chi}=\operatorname{dim}_{l} V(\chi)$. Then there exists an l-basis

$$
\Delta=\left\{e_{j}^{\chi} \mid \chi \in \Lambda, j=1, \ldots, d_{\chi}\right\}
$$

of $V_{l}$ such that $\gamma e_{j}^{\chi}=e_{j}^{\gamma \chi}$ for every $\gamma \in \Gamma$.

Proof. We may assume that $\Gamma$ acts transitively on $\Lambda$. Then $d=\operatorname{dim}_{l} V(\chi)$ is independent of $\chi \in \Lambda$.

Choose a weight $\chi_{0} \in \Lambda$. The stabilizer $\Gamma_{0}$ of $\chi_{0}$ in $\Gamma$ acts semi-linearly on the $l$-vector space $V\left(\chi_{0}\right)$. By the no-name lemma [Sh, Appendix 3] there exists a basis $e_{1}, \ldots, e_{d}$ of $V\left(\chi_{0}\right)$ such that each $e_{i}$ is preserved by $\Gamma_{0}$. Now for $\chi \in \Lambda$ and $j=1, \ldots, d$, set $e_{j}^{\chi}:=\gamma\left(e_{j}\right)$, where $\gamma \in \Gamma$ takes $\chi_{0}$ to $\chi$. It is now easy to see that the $e_{j}^{\chi}$ are well defined and form an $l$-basis of $V_{l}$ with the desired property.

Corollary 2.4. Suppose $A$ is split by a Galois extension $l / k$ and $\psi$ is an irreducible representation of $A$. Then $\operatorname{dim} \psi$ divides $[l: k]$.

Proof. By our construction $\Gamma=\operatorname{Gal}(l / k)$ permutes the $l$-basis $\Delta$ of $V_{l}$. Since $V$ is $k$-irreducible, this permutation action is transitive. Hence, $|\Delta|=\operatorname{dim} \psi$ divides $|\Gamma|=[l: k]$.

Now consider the $k$-torus $T:=\operatorname{Diag}(\mathbb{Z}[\Delta])$, which is split over $l$ and quasi-split over $k$. By our construction $T$ is equipped with a representation

$$
\iota: T \hookrightarrow \mathrm{GL}(V) \text {. }
$$


In the basis $\Delta$ of $V_{l}$, this representation is given by $\iota(t) \cdot e_{j}^{\chi}=\chi(t) e_{j}^{\chi}$. Note that by Galois descent, $\iota$ is defined over $k$. One easily checks that $\iota$ is generically free (this can be done over $l$ ).

We also remark that the original representation $\psi: A \rightarrow \mathrm{GL}(V)$ can be written as a composition $\psi=\iota \circ \hat{\psi}$, where $\hat{\psi}: A \rightarrow T$ is induced by the map $\mathbb{Z}[\Delta] \rightarrow X(A)$ of $\Gamma$-modules, sending $e_{j}^{\chi}$ to $\chi$.

Lemma 2.5. Every faithful representation $\psi: A \rightarrow \mathrm{GL}(V)$ of $A$ is generically free.

Proof. As we saw above, $\psi=\iota \circ \hat{\psi}$, where $\iota: T \rightarrow \mathrm{GL}(V)$ is generically free. If $\psi$ is faithful then $\hat{\psi}: A \rightarrow T$ is faithful, and hence, $\psi$ is generically free.

Lemma 2.6. Let $N$ be a closed subgroup of $A, l / k$ be a Galois splitting field of $A$ and $\Gamma=\operatorname{Gal}(l / k)$. Then

$$
\min \operatorname{dim} \psi=\min \operatorname{rank}(P)
$$

where the minimum on the left hand side is taken over all $k$-representations $\psi$ of $A$ with kernel $N$, and the minimum on the right is taken over all homomorphisms $f: P \rightarrow X(A)$ of $\mathbb{Z} \Gamma$-modules, with $P$ permutation and $\operatorname{cokernel}(f)=X(N)$.

Proof. Given $\psi: A \rightarrow \mathrm{GL}(V)$ with kernel $N$, write $\psi: A \stackrel{\hat{\psi}}{\rightarrow} T \stackrel{\iota}{\hookrightarrow} \mathrm{GL}(V)$ as above, where $T$ is a quasi-split $k$-torus of dimension $\operatorname{dim} T=\operatorname{rank} X(T)=$ $\operatorname{dim} \psi$ which splits over $l$. Then $\operatorname{ker} \hat{\psi}=N$ and the cokernel of the induced map $X(\hat{\psi}): X(T) \rightarrow X(A)$ of $\mathbb{Z} \Gamma$-modules is $X(N)$.

Conversely, if $P$ is a permutation $\mathbb{Z} \Gamma$-module then we can embed the torus $\operatorname{Diag}(P)$ in $\mathrm{GL}_{n}$, where $n=\operatorname{rk} P\left[\mathrm{Vos}_{2}\right.$, Section 6.1]. A map $f: P \rightarrow$ $X(A)$ of $\mathbb{Z} \Gamma$-modules with cokernel $X(N)$ then yields a representation $A \rightarrow$ $\operatorname{Diag}(P) \hookrightarrow \mathrm{GL}_{n}$ with kernel $N$.

\section{A LOWER BOUND ON ESSENTIAL $p$-DIMENSION}

Consider an exact sequence of algebraic groups over $k$

$$
1 \rightarrow C \rightarrow G \rightarrow Q \rightarrow 1
$$

such that $C$ is central in $G$ and isomorphic to $\mu_{p}^{r}$ for some $r \geq 0$. Given a character $\chi: C \rightarrow \mu_{p}$, we will, following [KM], denote by $\operatorname{Rep}^{\chi}$ the set of irreducible representations $\phi: G \rightarrow \mathrm{GL}(V)$, such that $\phi(c)=\chi(c)$ Id for every $c \in C$.

Theorem 3.1. Suppose a sequence of $k$-groups of the form (3) satisfies the following condition:

$$
\operatorname{gcd}\left\{\operatorname{dim}(\phi) \mid \phi \in \operatorname{Rep}^{\chi}\right\}=\min \left\{\operatorname{dim}(\phi) \mid \phi \in \operatorname{Rep}^{\chi}\right\}
$$

for every character $\chi: C \rightarrow \mu_{p}$. Then

$$
\operatorname{ed}(G ; p) \geq \min \operatorname{dim}(\psi)-\operatorname{dim} G,
$$


where the minimum is taken over all finite-dimensional representations $\psi$ of $G$ such that $\psi_{\mid C}$ is faithful.

Proof. Denote by $C^{*}:=\operatorname{Hom}\left(C, \mu_{p}\right)$ the character group of $C$. Let $V$ be a generically free $Q$-module, and $U \subseteq V$ an open dense $Q$-invariant subvariety such that $U \rightarrow U / Q$ is a $Q$-torsor. Then let $E \rightarrow \operatorname{Spec} K$ be the generic fibre of this torsor, and let $\beta: C^{*} \rightarrow \operatorname{Br}_{p}(K)$ denote the homomorphism that sends $\chi \in C^{*}$ to the image of $E \in H^{1}(K, Q)$ in $\operatorname{Br}_{p}(K)$ under the map

$$
H^{1}(K, Q) \rightarrow H^{2}(K, C) \stackrel{\chi_{*}}{\rightarrow} H^{2}\left(K, \mu_{p}\right)=\mathrm{Br}_{p}(K)
$$

given by composing the connecting map with $\chi_{*}$. Then there exists a basis $\chi_{1}, \ldots, \chi_{r}$ of $C^{*}$ such that

$$
\operatorname{ed}(G ; p) \geq \sum_{i=1}^{r} \operatorname{ind} \beta\left(\chi_{i}\right)-\operatorname{dim} G
$$

see $\left[\mathrm{Me}_{1}\right.$, Theorem 4.8, Example 3.7]. Moreover, by [KM], Theorem 4.4, Remark 4.5]

$$
\operatorname{ind} \beta\left(\chi_{i}\right)=\operatorname{gcd} \operatorname{dim}(\psi) \text {, }
$$

where the greatest common divisor is taken over all (finite-dimensional) representations $\psi$ of $G$ such that $\psi_{\mid C}$ is scalar multiplication by $\chi_{i}$. By our assumption, gcd can be replaced by min. Hence, for each $i \in\{1, \ldots, r\}$ we can choose a representation $\psi_{i}$ of $G$ with

$$
\operatorname{ind} \beta\left(\chi_{i}\right)=\operatorname{dim}\left(\psi_{i}\right)
$$

such that $\left(\psi_{i}\right)_{\mid C}$ is scalar multiplication by $\chi_{i}$.

Set $\psi:=\psi_{1} \oplus \cdots \oplus \psi_{r}$. The inequality (4) can be written as

$$
\operatorname{ed}(G ; p) \geq \operatorname{dim}(\psi)-\operatorname{dim} G \text {. }
$$

Since $\chi_{1}, \ldots, \chi_{r}$ form a basis of $C^{*}$ the restriction of $\psi$ to $C$ is faithful. This proves the theorem.

\section{Proof of the MAin Result}

The following lemma generalizes [MR, Lemma 4.1].

Lemma 4.1. Let $A$ be an algebraic group of multiplicative type over a field $k$, and let $B \subset A$ a closed subgroup of (finite) index prime to $p$. Then $\operatorname{ed}(A ; p)=\operatorname{ed}(B ; p)$.

Proof. The inequality ed $(B ; p) \leq \operatorname{ed}(A ; p)$ is clear, since $\operatorname{dim} A=\operatorname{dim} B$; see $\mathrm{Me}_{1}$, Corollary 4.3].

To prove the opposite inequality, set $Q:=A / B$. In view of the exact sequence $H^{1}(K, B) \rightarrow H^{1}(K, A) \rightarrow H^{1}(K, Q)$ it suffices to show that every $Q$-torsor $X \rightarrow \operatorname{Spec}(K)$ splits over a finite prime to $p$ extension of $K$. (Here $K$ is assumed to be an arbitrary field extension of $k$.)

First suppose char $k=p$. In this case $X$ is étale over $\operatorname{Spec}(K)$ (since $Q$ is étale over $\operatorname{Spec}(K)$, see [Wa, 14.4]). The proof now proceeds as in [MR, 
Lemma 4.1]. That is, $X$ is $K$-isomorphic to a direct product $\operatorname{Spec}\left(K_{1} \times \cdots \times\right.$ $K_{n}$ ), where each $K_{i} / K$ is a finite separable field extension. One of the fields $K_{i}$ has degree prime to $p$ over $K$, and we get a $K_{i}$-point of $X$ from the map $\operatorname{Spec}\left(K_{i}\right) \rightarrow X$, induced by the projection $K[X] \rightarrow K_{i}$. This implies that $X$ splits over $K_{i}$.

Now suppose char $k \neq p$. By [EKM, Prop 101.16] there exists an algebraic field extension $K^{(p)} / K$ such that every finite extension of $K^{(p)}$ has degree a power of $p$ and every finite sub-extension $L / K$ of $K^{(p)} / K$ has degree prime to $p$. It is easy to see that $K^{(p)}$ is a perfect field and $\Gamma=\operatorname{Gal}\left(K_{\text {alg }} / K^{(p)}\right)$ is a profinite $p$-group. Since $Q\left(K_{\text {alg }}\right)$ has order prime to $p$ the group $H^{1}\left(K^{(p)}, Q\right)=H^{1}\left(\Gamma, Q\left(K_{\text {alg }}\right)\right)$ is trivial by $\left[\mathrm{Se}_{2}\right.$, I.5, ex. 2]. Thus $X$ splits over $K^{(p)}$ and hence over a finite sub-extension $L / K$ of $K^{(p)} / K$.

Proposition 4.2. Let $G$ be an algebraic group of multiplicative type over $k, T$ its maximal $k$-torus, and $l / k$ a minimal Galois splitting field of $T$. Let $N \subset G$ be a finite $k$-subgroup whose order is coprime to both $[l: k]$ and $|G / T|$. Let $\pi: G \rightarrow G / N$ be the natural projection. Then

$$
\pi_{*}: H^{1}(K, G) \rightarrow H^{1}(K, G / N)
$$

is bijective, for any field extension $K / k$. In particular, $\operatorname{ed}(G)=\operatorname{ed}(G / N)$.

The following argument, simplifying our earlier proof, was suggested to us by Merkurjev.

Proof. We claim that $H^{1}(K, G)$ is $m$-torsion, where $m=[l: k] \cdot|G / T|$. Indeed, since $T_{K}$ is split by a Galois extension of degree dividing $[l: k]$, restricting and corestricting in Galois cohomology yields $[l: k] \cdot H^{1}(K, T)=$ (0). On the other hand, since $|G / T| \cdot H^{1}(K, G / T)=(0)$, the exact sequence

$$
H^{1}(K, T) \rightarrow H^{1}(K, G) \rightarrow H^{1}(K, G / T)
$$

shows that $H^{1}(K, G)$ is $m$-torsion, as claimed. Note that $N$ is contained in $T$ and the quotient of $G / N$ by its maximal torus $T / N$ is isomorphic to $G / T$. So the group $H^{1}(K, G / N)$ is $m$-torsion as well.

Now let $n=|N|$ and $p_{n}: G \rightarrow G$ be given by $g \rightarrow g^{n}$. The induced map $H^{1}(K, G) \stackrel{\left(p_{n}\right)_{*}}{\longrightarrow} H^{1}(K, G)$ is multiplication by $n$. Since $H^{1}(K, G)$ is $m$-torsion and by assumption $n$ and $m$ coprime, $\left(p_{n}\right)_{*}$ is an isomorphism. Moreover, $N$ lies in the kernel of $p_{n}$ and so $\left(p_{n}\right)_{*}$ factors through $\pi_{*}$ :

$$
\left(p_{n}\right)_{*}: H^{1}(K, G) \stackrel{\pi_{*}}{\rightarrow} H^{1}(K, G / N) \rightarrow H^{1}(K, G) .
$$

In particular, $\pi_{*}$ is injective. A similar argument shows that composing these maps in the opposite order,

$$
H^{1}(K, G / N) \rightarrow H^{1}(K, G) \stackrel{\pi_{*}}{\rightarrow} H^{1}(K, G / N),
$$

we get an isomorphism as well. This shows that $\pi_{*}$ is surjective and hence, bijective, as desired. 
Proof of the Theorem 1.1. We will first prove $\operatorname{ed}(G ; p) \geq \min \operatorname{dim}(\psi)-\operatorname{dim} G$, where the minimum is over $p$-faithful representations. Since $G$ is split by a Galois extension of $p$-power degree, Corollary 2.4 tells us that for any character $\chi$ of $C(G)$ and any $\phi \in \operatorname{Rep}^{\chi}, \operatorname{dim}(\phi)$ is a power of $p$. By Theorem 3.1. $\operatorname{ed}(G ; p) \geq \min \operatorname{dim}(\psi)-\operatorname{dim} G$, where $\psi$ ranges over representations of $G$ whose restriction to $C(G)$ is faithful. By Lemma 2.2 representations with this property are precisely the $p$-faithful representations.

We will now show that $\operatorname{ed}(G ; p) \leq \operatorname{dim} \psi-\operatorname{dim} G$ for any $p$-faithful representation $\psi$ of $G$. We will proceed in two steps.

Step 1 . Suppose $G$ is an extension of a $p$-group $F$ by a torus $T$. Since $N:=$ $\operatorname{ker} \psi$ is finite of order prime to $p$, Proposition 4.2 yields $\operatorname{ed}(G)=\operatorname{ed}(G / N)$. Now $\psi$ can be considered as a faithful representation of $G / N$. By Lemma 2.5. this representation of $G / N$ is generically free. By (2),

$$
\operatorname{ed}(G ; p) \leq \operatorname{ed}(G)=\operatorname{ed}(G / N) \leq \operatorname{dim} \psi-\operatorname{dim}(G / N)=\operatorname{dim} \psi-\operatorname{dim}(G),
$$

as desired.

Taking $\psi$ to be of minimal dimension, we also see that in this case we have $\operatorname{ed}(G ; p)=\operatorname{ed}(G)$, as asserted in the statement of the theorem.

Step 2. Let $G$ be an arbitrary group of multiplicative type. Let $T$ be the maximal torus of $G$, and $F^{\prime}$ be the Sylow $p$-subgroup of the multiplicative finite group $F:=G / T$. Recall that $F^{\prime}$ is defined as $\operatorname{Diag}(X(F) / Y)$, where $Y$ is the submodule of elements of order prime to $p$.

Now denote the preimage of $F^{\prime}$ under the projection $G \rightarrow F=G / T$ by $G^{\prime}$. Since $G^{\prime}$ is an extension of a $p$-group by a torus, we know from Step 1 that

$$
\operatorname{ed}\left(G^{\prime} ; p\right) \leq\left.\operatorname{dim} \psi\right|_{G^{\prime}}-\operatorname{dim} G^{\prime}=\operatorname{dim} \psi-\operatorname{dim} G .
$$

The index of $G^{\prime}$ in $G$ is finite and prime to $p$, hence $\operatorname{ed}(G ; p)=\operatorname{ed}\left(G^{\prime} ; p\right)$ by Lemma 4.1 and the desired inequality, $\operatorname{ed}(G ; p) \leq \operatorname{dim} \psi-\operatorname{dim} G$ follows.

\section{Main theOrem in the language of Character modules}

Let $G$ be of multiplicative type over $k$ and let $l / k$ be a Galois splitting field of $G$. We will call a map of $\mathbb{Z} \operatorname{Gal}(l / k)$-modules $P \rightarrow X(G)$ a $p$-presentation if $P$ is permutation, and the cokernel is finite of order prime to $p$.

We now restate our Theorem 1.1 in a way that is often more convenient to use.

Corollary 5.1. Let $G$ be a group of multiplicative over $k, l / k$ be a finite Galois splitting field of $G$, and $\Gamma_{p}$ be a Sylow p-subgroup of $\operatorname{Gal}(l / k)$. Then

$$
\operatorname{ed}(G ; p)=\min r k \operatorname{ker} \phi
$$

where the minimum is taken over all p-presentations $\phi: P \rightarrow X(G)$ of $X(G)$, viewed as a $\mathbb{Z} \Gamma_{p}$-module.

Proof. Let $k^{\prime}=l^{\Gamma_{p}}$. Then $\operatorname{Gal}\left(l / k^{\prime}\right)=\Gamma_{p}$. Since $\left[k^{\prime}: k\right]$ is finite and prime to $p$, (1) tells us that $\operatorname{ed}(G ; p)=\operatorname{ed}\left(G_{k^{\prime}} ; p\right)$. By Theorem $1.1 \operatorname{ed}\left(G_{k^{\prime}} ; p\right)=$ 
$\min \operatorname{dim}(\psi)-\operatorname{dim} G$, where the minimum is taken over all $p$-faithful representations $\psi$ of $G_{k^{\prime}}$. By Lemma 2.6

$$
\min \operatorname{dim}(\psi)-\operatorname{dim} G=\min \operatorname{rank}(P)-\operatorname{dim} G=\min r k \operatorname{ker} \phi,
$$

where the minimum on the right is taken over all $p$-presentations $\phi: P \rightarrow$ $X(G)$, as in the statement of the theorem.

Example 5.2. Let $T$ be a torus of dimension $<p-1$. Then $\operatorname{ed}(T ; p)=0$, because there is no non-trivial integral representation of dimension $<p-1$ of any $p$-group [AP, Satz].

Example 5.3. Assume char $k=0$, and let $\Gamma=\mathcal{S}_{p^{r}}$ denote the symmetric group for some $r \geq 1$. The generic torus $T$ of $\mathrm{PGL}_{n}$, defined in $\mathrm{Vos}_{2}, \S 4.1-$ 4.2 ], is of dimension $p^{r}-1$ and has character lattice

$$
X(T)=\left\{a \in \mathbb{Z}^{p^{r}} \mid a_{1}+\cdots+a_{p^{r}}=0\right\}
$$

with the natural action of $\Gamma$ on it; see $\operatorname{Vos}_{1}$. Let $\Gamma_{p}$ be a Sylow $p$-subgroup of $\Gamma$. In [MR, Prop. 7.2] it is shown that the minimal rank of a permutation module with a $p$-presentation to $X(T)$ is $p^{2 r-1}$. Thus by Corollary 5.1 , $\operatorname{ed}(T ; p)=p^{2 r-1}-p^{r}+1$.

\section{Forms of $\mu_{n}$}

Proposition 6.1. Let $A$ be a twisted form of $\mu_{p^{n}}$ over $k$ and $l / k$ a minimal Galois splitting field. Then $\operatorname{ed}(A ; p)=p^{r}$, where $p^{r}$ is the highest power of $p$ dividing $[l: k]$.

Proof. Let $\Gamma_{p}$ be a Sylow $p$-subgroup of $\operatorname{Gal}(l / k)$ and $\phi: P \rightarrow X(A)$ be a $p$ presentation. Since $\phi$ has prime to $p$ cokernel and $X(A)$ is a cyclic $p$-group, $\phi$ must be surjective. Thus, if $\Lambda$ is a basis of $P$, permuted by $\Gamma_{p}$, some element $\lambda \in \Lambda$ maps to a generator $a$ of $X(A)$. Moreover, $\Gamma_{p}$ acts faithfully on $X(A)$ and $|\Lambda| \geq\left|\Gamma_{p} \lambda\right| \geq\left|\Gamma_{p} a\right|=\left|\Gamma_{p}\right|$. Conversely we have a surjective homomorphism $\mathbb{Z}\left[\Gamma_{p} a\right] \rightarrow X(A)$ that sends $a$ to itself. So the minimal value of $\operatorname{rk} P$ is $\left|\Gamma_{p}\right|$. Now apply Corollary 5.1 .

Remark 6.2. For char $k \neq p$, Proposition 6.1 was previously known in the following special cases:

For twisted cyclic groups of order 4 it is due to M. Rost $[\mathrm{Ro}]$ and in the case of cyclic groups of order 8 to G. Bayarmagnai Ba. The case of constant cyclic groups of arbitrary prime power order is due to M. Florence [Fl].

Example 6.3. Let char $k=p$. D. Tossici and A. Vistoli [TV, Question 4.1 (2)] asked if the essential dimension of every algebraic $k$-group of order $p^{n}$ is $\leq n$. The following example, with $n=2$ and $p>2$, answers this question in the negative.

Let $l / k$ be a cyclic extension of order $p$; set $\Gamma:=\operatorname{Gal}(l / k)$. (For example, we can take $k$ and $l$ to be finite fields of orders $p$ and $p^{p}$, respectively.) Now let $M \simeq \mathbb{Z} / p^{2} \mathbb{Z}$ be the $\Gamma$-module obtained by identifying $\Gamma$ with the unique subgroup of $\operatorname{Aut}\left(\mathbb{Z} / p^{2} \mathbb{Z}\right) \simeq \mathbb{Z} / p(p-1) \mathbb{Z}$ of order $p$. By construction $G=$ 
$\operatorname{Diag}(M)$ is a form of $\mu_{p^{2}}$ defined over $k$, whose minimal Galois splitting field is $l$. Proposition 6.1 now tells us that $\operatorname{ed}(G)=\operatorname{ed}(G ; p)=[l: k]=p>2$.

\section{TWISTED $p$-GROUPS}

In this section we will use Theorem 3.1 to generalize the KarpenkoMerkurjev theorem to arbitrary (possibly twisted) finite $p$-groups over a field $k$, assuming that char $k \neq p$ and $k$ contains a primitive $p$ th root of unity.

Theorem 7.1. Let $G$ be an algebraic group over $k$ such that $G_{L}$ is a constant group of order $p^{n}$ for some $n \geq 1$ and some Galois extension $L / k$ of $p$-power degree. Then

$$
\operatorname{ed}(G)=\operatorname{ed}(G ; p)=\min \operatorname{dim} \psi,
$$

where $\psi$ runs through all faithful representations of $G$.

Proof. The inequalities ed $(G ; p) \leq \operatorname{ed}(G) \leq \min \operatorname{dim} \psi$ follow from (2). Hence it suffices to show that $\operatorname{ed}(G ; p) \geq \min \operatorname{dim} \psi$.

Since char $k \neq p$ the centre of $G$ is of multiplicative type, the subgroup $C(G)=\operatorname{Split}_{k}(Z(G)[p]$ ) is well-defined (as in Section 21) and is isomorphic to $\mu_{p}^{r}$ for some $r \geq 1$.

We claim that every irreducible representation $\psi$ of $G$ has dimension equal to a power of $p$. Denote by $\zeta$ a primitive root of unity of order equal to the exponent of $G(L)$. Since $k$ contains a primitive $p$ th root of unity, $L^{\prime}:=L(\zeta)$ is Galois over $k$ and of $p$-power degree, and $\psi$ decomposes over $L^{\prime}$ as a direct sum of absolutely irreducible representations of the abstract $p$-group $G\left(L^{\prime}\right)=G(L)$. All direct summands in this decomposition have the same dimension, equal to a power of $p$. By [Ka, Theorem 5.22] the number of direct summands in this decomposition is also a power of $p$, and the claim follows.

Therefore, Theorem 3.1 can be applied, i.e., $\operatorname{ed}(G ; p) \geq \min \operatorname{dim} \psi$ taken over all representations $\psi$ of $G$ whose restriction to $C(G)$ is faithful. Let $N$ be the kernel of such a representation. We claim that $N \cap C(G)=\{1\}$ implies that $N$ is trivial. If $G$ is constant we have $C(G)=Z(G)[p]$ since $k$ contains a primitive $p$ th root of unity and the claim is a standard elementary fact about $p$-groups. The general case follows from Lemma 2.1 applied to $A=Z(G)[p] \cap N$.

Remark 7.2. Theorem 7.1 allows one to compute ed $(G ; p)$, at least in principle, for any étale algebraic group $G$ over $k$, provided $\operatorname{char}(k) \neq p$.

To carry out this computation, we first pass to a suitable Galois extension $L / k$ of degree prime to $p$ such that $L$ contains a primitive $p$ th root of unity and $G_{L}$ becomes constant over a Galois extension $E / L$ of $p$-power degree.

We claim that $G_{L}$ has a Sylow $p$-subgroup $S$ defined over $L$. Indeed, the $p$-group $\operatorname{Gal}(E / L)$ permutes the Sylow subgroups of $G(E)$. By the Sylow theorems, the number of such subgroups is prime to $p$. Thus one of them is fixed by the $p$-group $\operatorname{Gal}(E / L)$. This proves the claim. 
Now we have $\operatorname{ed}(G ; p)=\operatorname{ed}\left(G_{L} ; p\right)=\operatorname{ed}(S ; p)$, and $\operatorname{ed}(S ; p)$ is given by Theorem 7.1,

\section{ACKNowledgments}

The authors are grateful to A. S. Merkurjev for numerous constructive comments on earlier versions of this paper, and to A. Auel and A. Vistoli for helpful discussions.

\section{REFERENCES}

[AP] H. Abold, W. Plesken, Ein Sylowsatz für endliche $p$-Untergruppen von $\operatorname{GL}(n, Z)$, Math. Ann. 232 (1978), no. 2, 183-186.

[Ba] G. Bayarmagnai, Essential dimension of some twists of $\mu_{p^{n}}$, Proc. Symp. Algebraic Number Theory and Related Topics, 145-151, RIMS Kôkyûroku Bessatsu, B4, Res. Inst. Math. Sci. (RIMS), Kyoto (2007).

[BF] G. Berhuy, G. Favi, Essential Dimension: A Functorial Point of View (after A. Merkurjev), Doc. Math. 8 (2003), 279-330.

[BM] S. Baek, A. Merkurjev, Essential dimension of central simple algebras, preprint, http://www.math.ucla.edu/ merkurev/publicat.htm

[BR] J. Buhler, Z. Reichstein, On the essential dimension of a finite group, Compositio Mathematica 106:159-179 (1997).

[EKM] R. Elman, N. Karpenko, A. Merkurjev: The algebraic and geometric theory of. quadratic forms. Amer. Math. Soc. Coll. Publ. 56, Providence, RI: Amer. Math. Soc. (2008).

[Fl] M. Florence, On the essential dimension of cyclic p-groups, Inventiones Mathematicae, 171 (2007), 175-189.

[Ka] G. Karpilovsky, Clifford Theory for Group Representations. Mathematics Studies, 156. North-Holland, Netherlands, (1989).

[KM] N. Karpenko, A. Merkurjev, Essential dimension of finite p-groups, Inventiones Mathematicae, 172 (2008), 491-508.

$\left[\mathrm{Me}_{1}\right]$ A. Merkurjev, Essential dimension, in Quadratic forms - algebra, arithmetic, and geometry (R. Baeza, W.K. Chan, D.W. Hoffmann, and R. Schulze-Pillot, eds.), Contemporary Mathematics 493 (2009), 299-326.

$\left[\mathrm{Me}_{2}\right]$ A. Merkurjev, A lower bound on the essential dimension of simple algebras, preprint, http://www.math.ucla.edu/ merkurev/publicat.htm

[MR] A. Meyer, Z. Reichstein, The essential dimension of the normalizer of a maximal torus in the projective linear group, Algebra and Number Theory, 3, no. 4 (2009), 467-487.

[Re] Z. Reichstein, On the Notion of Essential Dimension for Algebraic Groups, Transformation Groups, 5, 3 (2000), 265-304.

[RY] Z. Reichstein, B. Youssin, Essential Dimensions of Algebraic Groups and a Resolution Theorem for $G$-varieties, with an appendix by J. Kollar and E. Szabo, Canadian Journal of Mathematics, 52, 5 (2000), 1018-1056.

[Ro] M. Rost, Essential dimension of twisted $C_{4}$, preprint, http://www.math.uni-bielefeld.de/ rost/ed.html

$\left[\mathrm{Se}_{1}\right]$ J.-P. Serre, Linear representations of finite groups, Graduate Texts in Mathematics, 42, Springer-Verlag, 1977.

$\left[\mathrm{Se}_{2}\right]$ J.-P. Serre, Galois cohomology. Springer Monographs in Mathematics. SpringerVerlag, Berlin, 2002.

[Sh] I. R. Shafarevich, Basic Algebraic Geometry, vol. 1, 2nd edition, Springer-Verlag, 1994. 
[TV] D. Tossici, A. Vistoli, On the essential dimension of infinitesimal group schemes, preprint, http://www.mathematik.uni-bielefeld.de/lag/man/377

[Vos1] V. E. Voskresenskiu, Maximal tori without affect in semisimple algebraic groups, Mat. Zametki 44 (1988), no. 3, 309-318, 410. English transl.: Math. Notes 44 (1988) (1989), no. 3-4, 651-655.

[Vos 2$]$ V. E. Voskresenskiı̌, Algebraic Groups and Their Birational Invariants, American Mathematical Society, Providence, RI, 1998.

[Wa] W. C. Waterhouse, Introduction to affine group schemes. Springer-Verlag, New YorkBerlin, 1979. 